Jennifer King

George M. Holmes

Rebecca T. Slifkin

\section{Rural and Urban Differences in Children's Medicaid and CHIP Participation}

Efforts to increase enrollment in Medicaid and the Children's Health Insurance Program (CHIP) among uninsured children would benefit from an understanding of how program participation varies in rural and urban areas. Using Current Population Survey data from the period 2006-2007, rural participation rates were slightly higher than urban rates in the nation overall. There was no rural-urban difference when comparisons were based on within-state variation, independent of adjustment for individual characteristics. For researchers examining health policy issues strongly influenced by state policies or other state-level factors, this study highlights the challenges presented by national data sets with small or nonexistent samples from geographic areas within some states.

An ongoing goal of many state and federal policymakers is to expand health coverage among the uninsured, including the 7.3 million children who lacked health insurance in 2008 (DeNavas-Walt, Proctor, and Smith 2009). To achieve this goal, it will be necessary to find and enroll children who are eligible ${ }^{1}$ for public health insurance programs; it is estimated that from two-thirds to three-quarters of uninsured children are eligible for Medicaid or the Children's Health Insurance Program (CHIP) (Dubay, Holahan, and Cook 2006; Hudson and Selden 2007).

Efforts to boost Medicaid and CHIP participation received a renewed push from the Children's Health Insurance Program
Reauthorization Act of 2009. The act authorized new ways for states to identify and enroll eligible children and created "performance bonus" payments for states that meet federally defined targets for the number of children enrolled in Medicaid (Children's Health Insurance Program Reauthorization Act of 2009). Further, the legislation provided $\$ 80$ million in outreach grants to identify and enroll eligible children in Medicaid and CHIP; \$40 million was awarded to grantees in 41 states in the first round of funding in September 2009 (U.S. Department of Health and Human Services 2009).

In order for these efforts to be successful, it is important to understand how rates of program participation vary across rural and

Jennifer King is a doctoral student in the Department of Health Policy and Management and a predoctoral fellow at the Cecil G. Sheps Center for Health Services Research, University of North Carolina (UNC) at Chapel Hill. George M. Holmes, Ph.D., is a senior research fellow at the Cecil G. Sheps Center for Health Services Research at UNC. Rebecca T. Slifkin, Ph.D., is director of the North Carolina Rural Health Research and Policy Analysis Center at UNC's Cecil G. Sheps Center for Health Services Research. This work was funded through a cooperative agreement (\#2U1CRH03714-05-00) with the Office of Rural Health Policy, Health Resources and Services Administration, U.S. Department of Health and Human Services. Address correspondence to Ms. King at Cecil G. Sheps Center for Health Services Research, 725 Martin Luther King, Jr. Blvd., Campus Box 7590, Chapel Hill, NC 27599-7590. Email: jking@schsr.unc.edu 
urban areas. Public health insurance programs play an especially important role for rural residents, who have less access to employer-sponsored insurance than their urban counterparts (Lenardson et al. 2009). Medicaid and CHIP help fill this gap - the share of children insured by public programs is higher in rural areas than in urban areas (Lenardson et al. 2009). However, up-to-date information about Medicaid and CHIP participation rates - the share of children who are eligible for the programs and actually are enrolled - in rural areas specifically is scarce. This lack of research may be due in part to limitations of available national data on health insurance coverage and eligibility by rural residence.

To help inform efforts to increase Medicaid and CHIP participation, we used data from the Current Population Survey to examine whether the likelihood of participating in Medicaid/CHIP is different in rural and urban areas. Given data limitations associated with this research question, we also compared several analytic approaches and identified methodological issues that should be considered in future research.

\section{Background}

There is some evidence that Medicaid/CHIP participation rates historically have been lower in rural areas than urban areas in the nation overall. A study using survey data from 1999 found that rural children who were eligible for Medicaid or CHIP were about 10 percentage points less likely to be enrolled than their urban counterparts (Holahan, Dubay, and Kenney 2003). Further, an early CHIP evaluation found more confusion about eligibility rules among rural parents with uninsured children than urban parents (Kenney, Haley, and Blumberg 2002). However, some state-level studies found that, while controlling for other relevant factors, rural eligible children in California were more likely to be enrolled than their urban counterparts in 2001 (Kincheloe, Frates, and Brown 2007) and that rural residence had no effect on participation for children in Ohio in 1998 (McAlearney 2004).
There is reason to believe that national rural-urban patterns in participation rates may have changed during the past decade. States and other organizations have undertaken significant efforts to identify and enroll eligible children. Overall participation rates for CHIP have increased substantially, from less than $50 \%$ in 1998 to $66 \%$ to $69 \%$ in 2004 , while Medicaid participation rates increased from around $70 \%$ to nearly $80 \%$ during the time period (Selden, Hudson, and Banthin 2004; Dubay et al. 2007; Kenney and Cook 2007). At the same time, the share of rural children covered by public health insurance programs increased from about 20\% in 1997 to nearly $40 \%$ in 2005 , and uninsurance rates for rural children declined from 20\% in 1997 to $9 \%$ in 2005 (Ziller and Coburn 2009). To our knowledge, there are no published studies that investigate whether the rural-urban disparity in the likelihood that eligible children participate in Medicaid/CHIP has persisted amid these changes in children's insurance coverage rates.

Data limitations make it challenging to address this question. Analysis of enrollment rates requires detailed, nationally representative data on children's health insurance coverage and on factors that determine whether children qualify for Medicaid/CHIP (e.g., family structure and family income). The data must include information on rural residence and, because Medicaid/CHIP eligibility rules vary across states, the source must also identify the state in which each child lives. Only one publicly available data source meets these requirements: the Current Population Survey (CPS) conducted by the U.S. Census Bureau.

However, using the CPS to examine statelevel health insurance coverage presents some methodological challenges, including issues with sample size and sampling frame (Blewett and Davern 2006). Although the CPS is designed to produce state-level estimates for all 50 states, the sample size for some states can be small. This problem is amplified when examining subpopulations within states such as children eligible for public health insurance programs in rural and urban areas.

Further, although the sampling frame is designed to produce estimates that are 
representative of state populations on the whole, not all counties within a state are sampled. The finest measure of rural residence available on the CPS public use data is an indicator for whether individuals live in a metropolitan statistical area (MSA), as defined by the Office of Management and Budget's Core Based Statistical Area Designations. Nonmetropolitan samples in some states are quite small and in some cases nonexistent - in the 2008 and 2007 surveys, four states with nonmetropolitan counties had no CPS respondents from nonmetropolitan counties. (An additional two states plus the District of Columbia have no nonmetropolitan counties and therefore also have no nonmetropolitan sample.)

Despite these limitations, the CPS is the only publicly available data source that allows an examination of rural-urban statelevel patterns in children's public health insurance program participation.

\section{Data and Methods}

\section{Data}

The main data source for this analysis was the Annual Social and Economic Supplement (ASEC) of the CPS. The ASEC is a supplemental survey conducted by the CPS each March that collects detailed information on health insurance coverage and income in addition to employment and other issues. To obtain more precise estimates of the association between rural residence and Medicaid/CHIP participation, we pooled data from the 2007 and 2008 ASEC files.

Analysis sample. The analysis sample was composed of children who were eligible for Medicaid/CHIP and were either enrolled in one of the programs or were uninsured. To define the analysis sample, we took into account state-specific eligibility rules related to family size, income, age, and citizenship status. ${ }^{2}$ Medicaid and CHIP are operated as separate programs in many states. We took into account eligibility rules for both programs and included children in our analysis sample if they were qualified for either program. Because the CPS collects information that reflects respondents' circumstances in the prior year, we used state eligibility rules from 2006 and 2007.

Determining whether each child was eligible on the basis of income required four main steps. First, we grouped children into "budget units" composed of the individuals whose income would be counted in determining the child's eligibility. ${ }^{3}$ Second, we calculated total annual income within each budget unit. States have the flexibility to disregard portions of certain types of income (e.g., child support) in their eligibility determination procedures (Cohen Ross et al. 2008). We applied these "disregards" when calculating each budget unit's total income. Third, because income-eligibility thresholds are defined in terms of the federal poverty level and the federal poverty level varies by family size, we calculated each child's family size, counting the child, his or her parent(s) in the household, and siblings under 21 in the household. Finally, we compared total income for each child to state- and age-specific income thresholds to determine whether the child was eligible for Medicaid/CHIP.

We also took immigration status into account in determining whether children were eligible for Medicaid/CHIP. Undocumented immigrants do not qualify for federal funding under Medicaid or CHIP. Further, during the study period, federal funds could not be used to cover legal immigrants who had been in the United States for less than five years. However, several states covered immigrant children with state funds during this five-year window, and many of these state-funded programs did not distinguish program eligibility by documentation status ( $\mathrm{Ku}$ 2007; Hudson 2009). As discussed further in the limitations section, the CPS lacks data on the legal status of noncitizens. We classified noncitizen children as eligible for Medicaid/ CHIP if they met: a) income requirements, and $b$ ) the five-year residency requirement (if they lived in states that did not operate a state-funded program for immigrant children who were in the five-year window).

Finally, we automatically classified children in foster care and children receiving Supplemental Security Income (SSI) as eligible.

Rural residence. The key independent variable, rural residence, was a dichotomous 
variable indicating whether children lived in a nonmetropolitan county. Because of confidentiality restrictions, the metropolitan status indicator was suppressed for some observations on the ASEC public use files, including 440 observations in our analysis sample $(1 \%)$. These 440 observations came from four states; the remaining observations in these states were from metropolitan counties. Thus, as mentioned earlier, there were four states that did not have any sample identified as nonmetropolitan in the 2007 and 2008 ASEC even though the states contain nonmetropolitan counties. Further, there were eight additional states with very small samples (fewer than 100 observations) of our analysis population in nonmetropolitan or metropolitan areas. We conducted a number of analyses to determine the implications of an unidentified metropolitan status and small urban or rural samples within states for our results (described subsequently).

Dependent variable. The dependent variable was whether the child had any Medicaid/CHIP coverage in the year. The ASEC asks a series of questions about the types of health insurance coverage that each member of the household had at any point during the previous calendar year. There are separate questions about Medicaid and CHIP, and the interviewer uses the state's unique name for these programs when applicable. All children recorded as having Medicaid or CHIP coverage at some point during the year were given positive values for the outcome variable.

There are well-documented problems with self-reported data on Medicaid coverage in the CPS; estimates of Medicaid enrollment from the CPS are consistently lower than counts of enrollees from administrative data (Lewis, Elwood, and Czajka 1998; Hoffman and Holahan 2005; Call et al. 2008; Klerman et al. 2009). While there are legitimate reasons to expect survey data and administrative data to differ, there is evidence that a substantial portion of the discrepancy is due to reporting errors by CPS respondents (Davern et al. 2009). Research linking Medicaid administrative records to the CPS found that approximately $40 \%$ of children who were present in both the CPS and Medicaid administrative records failed to report Med- icaid coverage in the CPS; about $60 \%$ of these children were coded as having another type of insurance, while about $40 \%$ were coded as uninsured (derived from Table 2 in Davern et al. 2009). Further, the number of individuals who reported Medicaid coverage in the CPS but were not in Medicaid administrative records is nontrivial (Davern et al. 2009). To assess the extent to which this Medicaid measurement error may affect our estimates of the association between rural residence and Medicaid/CHIP participation, we conducted sensitivity analyses that partially adjust for the misreporting (Davern, Klerman and Ziegenfuss 2008). ${ }^{4}$

\section{Analytic Methods}

To examine the association between rural residence and Medicaid/CHIP participation, we estimated three logistic regression models that each approached the issue in a slightly different way. Because we were interested in Medicaid/CHIP participation among eligible children without other sources of insurance coverage, we conducted all analyses for children who were eligible for Medicaid/ CHIP and were either enrolled in the program at some point during the year or were uninsured for the entire year (e.g., eligible children who had private insurance coverage during the year were excluded).

First, we compared the probability of participation for all rural eligible children in the United States to the probability of participation for all urban eligible children. In the simple regression Model 1-the "simple difference" model- $\beta_{1}$ captures the difference between the probability of participation for eligible children in rural areas and the probability of participation for eligible children in urban areas:

\section{Model 1: $P($ Participation $)=f\left(\beta_{0}+\beta_{1}\right.$ Rural $)$}

Next, we evaluated whether there was a rural-urban difference in Medicaid/CHIP participation when using rural-urban comparisons based on children within the same state by including state fixed effects:

$$
\begin{aligned}
& \text { Model } 2: P(\text { Participation })= \\
& f\left(\alpha_{1} \text { Rural }+\alpha_{S} \text { State }\right)
\end{aligned}
$$


State was a set of 51 indicator variables for all states and the District of Columbia, and $\alpha_{1}$ captured the average within-state rural-urban difference in the probability of participation for eligible children. This model-the "withinstate" model-yields the average rural effect while adjusting for state-level effects.

The conclusions based on Model 1 and Model 2 could differ if states with higher (or lower) percentages of eligible children living in rural areas also had systematically higher (or lower) overall participation rates. The difference between the overall and withinstate comparisons has the potential to be amplified with the addition of states with no rural observations. States with no rural observations in the analysis population (either because the state has no nonmetropolitan counties or because the ASEC does not contain a nonmetropolitan sample in the state) will not contribute to the rural "effect" $\left(\alpha_{1}\right)$ in the state fixed-effects model. In order to understand the implications of limited rural or urban samples within states, we estimated Model 1 and Model 2 for three groups of states: all states $(50+$ DC); all states except those with nonmetropolitan counties but no nonmetropolitan sample $(46+$ DC); and all states except those with nonmetropolitan counties but fewer than 100 observations in either nonmetropolitan or metropolitan areas $\left(38+\right.$ DC). ${ }^{5}$

Finally, because there are differences in the characteristics of children who live in rural and urban areas and these characteristics may be associated with the likelihood that a child participates in Medicaid/CHIP, we also estimated rural and urban participation rates based on overall and within-state comparisons while controlling for children's individual and household characteristics.

\section{Model 3: $P($ Participation $)=$}

$$
f\left(\begin{array}{l}
\delta_{1} \text { Rural }+\delta_{s} \text { State }+ \\
\delta_{c} \text { IndividualCharacteristics }
\end{array}\right)
$$

IndividualCharacteristics was a set of individual and family characteristics likely to be associated with knowledge about Medicaid/CHIP, desire to enroll, and ability to apply.
Because the outcome variable was dichotomous, we estimated all models using logistic regression. All analyses were weighted, and we adjusted the standard errors of the logistic coefficient estimates for the complex survey design of the CPS (Davern et al. 2007). In addition to presenting coefficient estimates, we calculated the average incremental effects of rural residence and other individual characteristics on the probability of Medicaid/CHIP participation. For example, to obtain the average incremental effect of rural residence in Model 1, we generated two predicted probabilities of Medicaid/CHIP participation-one assuming all children lived in a rural area and one assuming all children lived in an urban area-and calculated the difference between the predicted probabilities. We created biascorrected $95 \%$ confidence intervals around all incremental effect estimates using Stata's bootstrap command with 500 repetitions.

To assess the robustness of our results, we also estimated several alternative model specifications. We estimated the models on an analysis sample of citizen children only. We estimated the models assuming all observations with unidentified metropolitan status were from nonmetropolitan areas and then assuming they were from metropolitan areas. Our substantive findings were consistent across these analyses. As discussed earlier, we also conducted analyses to assess the impact of Medicaid coverage misreporting in the CPS on estimates of rural-urban differences in Medicaid/CHIP participation.

\section{Results}

Characteristics of Children Eligible for Medicaid/CHIP in Rural and Urban Areas

Rural and urban eligible children were similar in terms of age, gender, and health status (Table 1). Eligible children in rural areas were substantially more likely to be white, nonHispanic compared to eligible urban children. The majority of eligible children lived in households where the highest educational attainment was a high school diploma (37\%) or some college or an associate's degree (32\%). Rural eligible children were slightly more likely to live in a household with at least a high school diploma than their urban coun- 
Table 1. Characteristics of children eligible for Medicaid/CHIP, 2006-2007

\begin{tabular}{lcrr}
\hline & Rural & Urban & Total \\
\hline Age (years) & 8.4 & 8.3 & 8.3 \\
Female (\%) & 49.0 & 49.1 & 49.1 \\
In fair/poor health (\%) & $5.0^{* *}$ & 3.7 & 3.9 \\
Has sibling in fair/poor health (\%) & 7.2 & 6.1 & 6.3 \\
Race (\%) & & & \\
White & $60.8^{* *}$ & 29.6 & 35.3 \\
Hispanic & $14.3^{* *}$ & 39.5 & 35.0 \\
Black & $17.2^{* *}$ & 24.0 & 22.8 \\
American Indian & $4.0^{* *}$ & .5 & 1.2 \\
Asian & $.5^{* *}$ & 3.5 & 3.0 \\
Multiple races & 3.2 & 2.7 & 2.8 \\
Highest education in household (\%) & & & \\
College degree & $8.1^{* *}$ & 11.0 & 32.4 \\
Some college or associate's degree & 34.0 & 31.8 & 36.9 \\
High school diploma or equivalent & $42.1^{* *}$ & 35.7 & 20.4 \\
Less than high school & $15.8^{* *}$ & 21.5 & \\
Income (\%) & & & 65.0 \\
Less than 100\% FPL & $68.0^{* *}$ & 64.3 & 31.3 \\
100\% to 199\% FPL & $29.4^{*}$ & 31.7 & 3.7 \\
200\% FPL or higher & $2.6^{* *}$ & 4.0 & 19.9 \\
Moved in last year (\%) & 21.1 & 19.6 & 50.4 \\
Survey year is 2008 (\%) & 50.0 & 50.5 & 38,220 \\
Sample size & 8,892 & 29,328 & \\
\hline
\end{tabular}

Note: Sample includes children under 19 who were eligible for Medicaid/CHIP and were either enrolled in Medicaid/CHIP or were uninsured.

$* *(*)$ Significantly different from urban at the .01(.05) level.

terparts. Children eligible for Medicaid/CHIP in rural areas also were slightly more likely than those in urban areas to have family incomes below the federal poverty level.

\section{Medicaid/CHIP Participation}

In Table 2, we present estimates of overall Medicaid/CHIP participation rates and findings from our "simple difference" and "within-state" models. About $73 \%$ of all children who were eligible for Medicaid/CHIP in 2006 or 2007 and had no other insurance coverage were enrolled in the programs (Group 1 in Table 2). The participation rate was slightly higher when states with rural areas but no rural sample were excluded and when states with rural areas but fewer than 100 observations in either rural or urban areas were excluded (Groups 2 and 3 in Table 2).

\section{Rural and Urban Differences in Medicaid/CHIP Participation}

When comparing rural eligible children to urban eligible children nationally, the prob- ability of participation was about three percentage points higher among rural children (Group 1 in Table 2). However, the rural-urban difference in Medicaid/CHIP participation based on within-state comparisons was not significantly different from zero.

The difference between the simple and within-state results for Group 1 was driven partly by the fact that the four states with nonmetropolitan counties but no nonmetropolitan sample had relatively low overall participation rates. Because they had no rural sample, these states contributed exclusively to the urban participation rate in the simple model for Group 1. When these four states were excluded from the analysis, the simple rural-urban difference was slightly lower in magnitude, but was still positive and statistically significant (Group 2 in Table 2). Because these four states did not contribute to the rural-urban difference in the state fixedeffects model, the within-state difference is identical for Groups 1 and 2. 


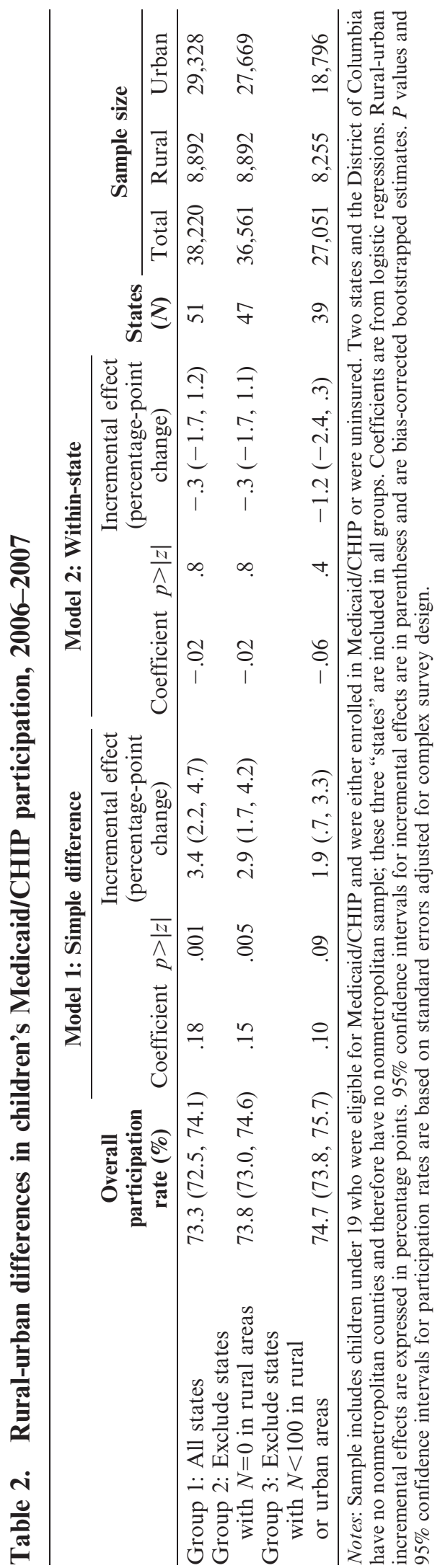

In the eight states with very small rural or urban samples (fewer than 100 observations), the rural participation rate was about five percentage points higher than the urban participation rate, but this estimate was imprecise and was not statistically significant (not shown). When these eight states also were excluded from the analysis, the simple rural-urban difference in participation rates was lower and hovered near statistical significance (the $p$ value of the logistic coefficient was .09 and the 95\% confidence interval around the incremental effect was .7 to 3.3 percentage points) (Group 3 in Table 2). The within-state difference was negative but not significant.

The null rural finding based on within-state comparisons for all states persisted after controlling for individual and household characteristics (Table 3). Many of the other characteristics in the model were significantly associated with participation. Being in fair or poor health or having siblings in fair or poor health was associated with an increased likelihood of being enrolled in Medicaid/ CHIP. Relative to eligible children who were white non-Hispanic, those who were black or multiracial were more likely to participate in Medicaid/CHIP, while Hispanic and Asian/ Pacific Islander children were less likely to participate. Eligible children with family incomes above the federal poverty level were less likely to be enrolled than eligible children in poorer families. Additionally, eligible children in the 2008 survey were slightly more likely to be enrolled than those in the 2007 survey.

\section{Discussion}

We did not find a substantial rural-urban difference in children's Medicaid/CHIP participation rates in the 2006-2007 period. Contrary to expectations, in the overall national comparison there was a statistically significant positive association between rural residence and participation (Model 1). However, the magnitude of the effect, about three percentage points, was relatively smallexpressed as a percentage difference from the global mean enrollment rate, the effect was only about 4\%. Moreover, we found no 
Table 3. Logistic regression of Medicaid/CHIP participation among eligible children, 2006-2007

\begin{tabular}{|c|c|c|c|}
\hline & Coefficient & $p>|z|$ & Incremental effect (percentage-point change) \\
\hline Rural & -.03 & .6 & $-.6(-2.1, .8)$ \\
\hline Age & -.05 & $<.001$ & $-1.0(-1.1,-.9)$ \\
\hline Female & .06 & .04 & $1.1(.1,2.1)$ \\
\hline In fair/poor health & .60 & $<.001$ & $9.6(6.7,11.8)$ \\
\hline Has sibling in fair/poor health & .51 & $<.001$ & $8.4(6.4,10.4)$ \\
\hline \multicolumn{4}{|l|}{ Race } \\
\hline Hispanic & -.26 & $<.001$ & $-4.8(-6.3,-3.3)$ \\
\hline Black & .15 & .02 & $2.6(.9,4.0)$ \\
\hline American Indian & -.30 & .1 & $-5.6(-9.6,-1.8)$ \\
\hline Asian & -.52 & $<.001$ & $-10.1(-13.2,-6.5)$ \\
\hline Multiple races & .29 & .02 & $4.8(1.8,7.2)$ \\
\hline \multicolumn{4}{|l|}{ Highest education in household } \\
\hline College degree & -.24 & .001 & $-4.5(-6.3,-2.9)$ \\
\hline Some college & .04 & .4 & $.7(-.7,1.8)$ \\
\hline Less than high school & -.06 & .4 & $-1.1(-2.3, .3)$ \\
\hline \multicolumn{4}{|l|}{ Income } \\
\hline $100 \%$ to $199 \%$ FPL & -.19 & $<.001$ & $-3.5(-4.5,-2.4)$ \\
\hline $200 \%$ FPL or higher & -.42 & $<.001$ & $-8.1(-10.9,-5.2)$ \\
\hline Moved in last year & -.03 & .6 & $-.6(-1.9, .8)$ \\
\hline Survey year is 2008 & .11 & .006 & $2.0(1.1,3.0)$ \\
\hline
\end{tabular}

Notes: Sample includes children under 19 who were eligible for Medicaid/CHIP and were either enrolled in Medicaid/CHIP or were uninsured $(N=38,220)$. Incremental effects are expressed in percentage points. $95 \%$ confidence intervals are in parentheses and are bias-corrected bootstrapped estimates. $P$ values are based on standard errors adjusted for complex survey design.

statistically significant rural-urban difference in Medicaid/CHIP participation in the nation overall based on within-state comparisons, independent of adjustment for individual characteristics (Models 2 and 3).

These results suggest progress has been made in equalizing rural and urban participation rates at the national level over the past decade. It is likely that state outreach and enrollment efforts, along with other trends, have contributed to these gains. Nonetheless, a substantial portion of children who were eligible for Medicaid/CHIP remained uninsured in both rural and urban areas. The Children's Health Insurance Program Reauthorization Act of 2009 includes promising avenues to reach these children, including the authorization of new outreach and enrollment approaches and increased funding to carry out these activities. It will be important for efforts to focus on children in both rural and urban areas. Further, policymakers may wish to consider subpopulations of eligible children who are at higher risk of going without coverage, including: older children,
Hispanic and Asian/Pacific Islander children (relative to white, non-Hispanic), and children in families with incomes above the poverty level. Similar risk factors also have been reported in previous research (Selden, Banthin, and Cohen 1998; Dubay, Kenney, and Haley 2002; McAlearny 2004; Kenney and Cook 2007).

The policy-relevant conclusions based on our results were similar across analytic approaches: there was not a large rural-urban disparity in Medicaid/CHIP participation. It is to be expected that the national-level ruralurban difference was not the same as the within-state comparison. This difference between models will remain as long as there is variation across states in the likelihood that eligible children actually enroll. While it is conceptually interesting to consider both the simple rural-urban difference in Medicaid/ CHIP participation and the difference based on within-state comparisons, many of the factors-and potential interventions - that affect the likelihood that an eligible child will enroll in Medicaid/CHIP exist at the state 
level (e.g., enrollment procedures and policies and local attitudes toward public assistance programs). Therefore, the models that examine rural-urban differences while holding the state environment fixed are especially useful. In this case, we found that much of the approximate three-percentage-point "rural advantage" was due to differences in statelevel factors associated with both the likelihood of participating in Medicaid/CHIP and the likelihood of living in a rural area; in other words, states with a larger proportion of eligible children in rural areas tended to have higher average participation rates.

It is also notable that the results were slightly different when states with small rural or urban samples were excluded from the analyses, underscoring the challenges of examining rural health issues that are strongly influenced by state policies or other state factors using existing national surveys. In this analysis, the magnitude of the simple ruralurban difference decreased as we excluded states with nonexistent or small rural samples. And, although the within-state estimates were not statistically significant for any of the three groups of states, the direction of the withinstate estimate changed when we excluded states with very small rural or urban samples. In some states with very small rural samples, rural participation rates were substantially higher than urban participation rates; however, the variance of these estimates was quite large because of the small sample size. It is customary to refrain from reporting state-level results based on small samples; it may also be important for rural health researchers to consider what effect small samples within states have on national-level results.

This study also highlights another related limitation of existing national data sources: we were unable to examine variation in children's public insurance program participation across the continuum of rural areas. Studies of insurance coverage have consistently found differences between rural counties adjacent to urban counties and more remote "nonadjacent" counties. Populations in nonadjacent counties tend to be poorer, are less likely to be privately insured, and are more likely to be uninsured or publicly insured than those in rural counties adjacent to an urban area or urban counties (Larson and Hill 2005; Lenardson et al. 2009; Ziller et al. 2008). Constrained by the sampling frame and limited access to geographic identifiers, we were only able to examine patterns in children's Medicaid/CHIP participation according to a dichotomous rural measure, which may obscure important differences across different types of rural areas.

Given the recent reauthorization of CHIP and ongoing efforts to expand health insurance coverage in the United States, the demand for improved local area information about public health insurance program participation is increasing. The Census Bureau's American Community Survey (ACS) is one potential source of such data. The ACS has a much larger sample than the CPS, and the ACS sample is drawn from all counties in the United States. The first health insurance estimates from the ACS were released in September 2009, and preliminary analyses indicate that national ACS estimates track closely with estimates from the CPS and other surveys (Turner, Boudreaux, and Lynch 2009). However, the health insurance questions on the ACS are less detailed - they do not use state-specific names for public programs, for example - and therefore may elicit less than ideal information about Medicaid and CHIP coverage specifically (Davern et al. 2009). Nonetheless, the ACS is a promising new source of data.

This analysis had limitations, including the potential that some key variables were measured with error. While we used detailed childand state-level information to determine which children were eligible for Medicaid/CHIP, we may have predicted state eligibility decisions imperfectly. Further, as noted earlier, there are well-documented problems with self-reported Medicaid coverage data in the CPS. Measurement error almost certainly exists for CHIP as well, but the nature and extent of this reporting error is not known. These potential sources of measurement error may have led to biased estimates of rural-urban differences in Medicaid/CHIP participation. However, our sensitivity analyses that partially adjusted for Medicaid misreporting bolster confidence in the substantive conclusions drawn from our results. 
As discussed previously, another limitation is that the CPS lacks information on the documentation status of noncitizens. The results presented here assume all noncitizens were legal residents and were eligible for Medicaid or CHIP if they met other eligibility requirements, including the five-year residency requirement in states that did not use state funds to cover legal residents in the country for shorter durations. This likely caused us to classify some immigrant children as eligible for Medicaid/CHIP when in fact their documentation status would have made them ineligible for the programs. However, in a sensitivity test that excluded all noncitizen children from the analysis, our substantive findings with regard to rural-urban differenc- es in Medicaid/CHIP participation did not change. ${ }^{6}$

This analysis provided updated information about rural-urban patterns in Medicaid/ CHIP participation for children and highlighted the difficulties associated with understanding geographic variation in participation rates using existing national data. As demand for local area data increases, it will be important to identify additional state and local sources of data and to invest in improved data collection at the national level. Identifying eligible uninsured children in both rural and urban areas and tailoring strategies to increase their Medicaid and CHIP participation will be a necessary component of efforts to cover all U.S. children.

\section{Notes}

1 The term "eligible" can have different meanings when referring to public health insurance programs. Research on this topic usually refers to individuals who are qualified to enroll in a public program as "eligible." Medicaid agencies, however, usually refer to enrolled individuals as "eligibles," meaning they are eligible to use services. Here, we use "eligible" to mean qualified to enroll.

2 We obtained information on states' income eligibility levels as of July 2006 and July 2007 from annual surveys conducted by the Center for Budget and Policy Priorities for the Kaiser Commission on Medicaid and the Uninsured (Cohen Ross, Horn, and Marks 2008; Cohen Ross, Cox, and Marks 2007). Information on disregards as of January 2008 was from Cohen Ross et al. (2008). Information on whether states used state-only funds to cover noncitizen children in Medicaid/CHIP during the five-year ban as of late 2006 was obtained from $\mathrm{Ku}$ (2007).

3 In general, Medicaid budget units for an unmarried child contain the child and his or her parent(s). For CHIP, budget unit rules can vary by state and sometimes also count income from other siblings under 21 or other family members in the household. For states that operate their CHIP programs as a Medicaid expansion, we used the Medicaid budget unit rules. For states with separate CHIP programs, we used state-specific CHIP budget rules based on information gathered from state web sites. For 10 states with separate CHIP programs, CHIP budget unit information could not be obtained; we used the Medicaid budget unit rules for these states. Results did not change when budget units were expanded to include siblings under 21 in these 10 states.

4 To investigate and address the Medicaid undercount in the CPS, Davern, Klerman, and Ziegenfuss (2008) linked 2001 and 2002 CPS data to administrative records from the Medicaid Statistical Information System (MSIS). Davern and colleagues published coefficient estimates from regressions of the likelihood that a given CPS record could be linked to MSIS data showing that a person was enrolled in Medicaid for at least one day during the year. To assess the possible impact of Medicaid coverage misreporting in the CPS on our estimates of rural-urban differences in Medicaid/CHIP participation, we applied these regression estimates to our CPS data to create a "partially adjusted" dependent variable - a continuous measure of the probability that MSIS data would indicate that each child in our sample was enrolled in Medicaid at some point during the year. Because this adjustment does not take CHIP reporting into account, we set the partially adjusted dependent variable equal to 1 for children who reported having CHIP coverage (i.e., we assume children who reported CHIP coverage had $100 \%$ probability of actually being enrolled in CHIP). Our analysis sample in the sensitivity analysis contained all children who were eligible for Medicaid/CHIP, including children who reported having insurance coverage aside from Medicaid/CHIP (since they may have a nonzero probability of being matched to administrative Medicaid data). We used ordinary least squares regression to estimate each of our three models twice, once with a dichotomous 
dependent variable equal to 1 if the child reported having Medicaid or CHIP coverage in the CPS and once with the partially adjusted dependent variable. For each of the three models, the substantive conclusions regarding rural residence were similar for the outcome variable based only on CPS reporting and the partially adjusted outcome variable. While this partial adjustment method has limitations (for a full discussion see Davern, Klerman, and Ziegenfuss 2008) and this sensitivity analysis sample is slightly different than the main analysis sample, this check bolsters confidence in the rural-urban patterns in the main analysis (which are not adjusted for Medicaid misreporting).

5 The states with nonmetropolitan counties but no nonmetropolitan sample were: Colorado, Louisiana, Nevada, and Utah. The additional states with nonmetropolitan counties but fewer than 100 observations in either nonmetropolitan or metropolitan areas were: Arizona, Florida, California, Connecticut, Maryland, Massachusetts, Montana, and Washington.

6 The simple rural-urban difference is slightly smaller (2.3 percentage points), but is still positive and statistically significant $(95 \% \mathrm{CI}$ : 1.3 to 3.6$)$.

\section{References}

Blewett, L. A., and M. Davern. 2006. Meeting the Need for State-Level Estimates of Health Insurance Coverage: Use of State and Federal Survey Data. Health Services Research 41(3):946-975.

Call, K. T., G. Davidson, M. Davern, L. A. Blewett, and R. Nyman. 2008. The Medicaid Undercount and Bias to Estimates of Uninsurance: New Estimates and Existing Evidence. Health Services Research 42(3):901-914.

Children's Health Insurance Program Reauthorization Act of 2009. Public Law 111-3. February 4 , 2009. Stat 123.8 .

Cohen Ross, D., A. Horn, R. Rudowitz, and C. Marks. 2008. Determining Income Eligibility in Children's Health Coverage Programs: How States Use Disregards in Children's Medicaid and CHIP. Washington, D.C.: Kaiser Commission on Medicaid and the Uninsured. www. kff.org/medicaid/7776.cfm. Accessed October 13, 2009.

Cohen Ross, D., A. Horn, and C. Marks. 2008. Health Coverage for Children and Families in Medicaid and SCHIP: State Efforts Face New Hurdles. Washington, D.C.: Kaiser Commission on Medicaid and the Uninsured. www.kff.org/ medicaid/7740.cfm. Accessed October 13, 2009.

Cohen Ross, D., L. Cox, and C. Marks. 2007. Resuming the Path to Health Coverage for Children and Parents: A 50-State Update on Eligibility Rules, Enrollment and Renewal Procedures, and Cost-Sharing Practices in Medicaid and SCHIP in 2006. Washington, D.C.: Kaiser Commission on Medicaid and the Uninsured. www.kff.org/medicaid/7608a.cfm. Accessed October 13, 2009.

DeNavas-Walt, C., B. D. Proctor, and J. C. Smith. 2009. Income, Poverty, and Health Insurance Coverage in the United States: 2008. Washington, D.C.: U.S. Census Bureau, Current Population Reports, P60-236. http://www. census.gov/prod/2009pubs/p60-236.pdf. Accessed September 15, 2009.
Davern, M., J. A. Klerman, D. K. Baugh, K. T. Call, and G. D. Greenberg. 2009. An Examination of the Medicaid Undercount in the Current Population Survey: Preliminary Results from Record Linking. Health Services Research 44(3):965-987.

Davern, M., B. C. Quinn, G. M. Kenney, and L. A. Blewett. 2009. The American Community Survey and Health Insurance Coverage Estimates: Possibilities and Challenges for Health Policy Researchers. Health Services Research 44(2, Part 1):593-605.

Davern, M., J. A. Klerman, and J. Ziegenfuss. 2008. Medicaid Under-Reporting in the Current Population Survey and One Approach for a Partial Correction. Minneapolis, Minn.: State Health Access Data Assistance Center. http://www.shadac.org/files/shadac/publications/ CPSMedicaid_Adj_Nov2008.pdf. Accessed October 1, 2009.

Davern, M., A. Jones, J. Lepkowski, G. Davidson, and L. A. Blewett. 2007. Estimating Regression Standard Errors with Data from the Current Population Survey's Public Use File. Inquiry 44(2):211-224.

Dubay, L., J. Holahan, and A. Cook. 2006. The Uninsured and the Affordability of Health Insurance Coverage. Health Affairs 26(1):w22-w30.

Dubay, L., J. Guyer, C. Mann, and M. Odeh. 2007. Medicaid at the Ten-Year Anniversary of SCHIP: Looking Back and Moving Forward. Health Affairs 26(2):370-381.

Dubay, L., G. Kenney, and J. Haley. 2002. Children's Participation in Medicaid and SCHIP: Early in the SCHIP Era. Assessing the New Federalism Policy Brief B-40. Washington, D.C.: Urban Institute. http://www. urban.org/publications/310430.html. Accessed October 13, 2009.

Hoffman, C., and J. Holahan. 2005. What is the Current Population Survey Telling Us about the Number of Uninsured? Washington, D.C.: 
Kaiser Commission on Medicaid and the Uninsured. http://www.kff.org/uninsured/7384. cfm. Accessed October 13, 2009.

Holahan, J., L. Dubay, and G. M. Kenney. 2003. Which Children Are Still Uninsured and Why? The Future of Children 13(1):55-79.

Hudson, J. L., and T. M. Selden. 2007. Children's Eligibility and Coverage: Recent Trends and a Look Ahead. Health Affairs 26(5):w618-w629.

Hudson, J. L. 2009. Families with Mixed Eligibility for Public Coverage: Navigating Medicaid, CHIP, and Uninsurance. Health Affairs 28(4):w697-w709. Online Data Supplement Appendix http://content.healthaffairs.org/cgi/ content/full/hlthaff.28.4.w697/DC2. Accessed September 29, 2009.

Kenney, G., and A. Cook. 2007. Coverage Patterns among SCHIP-Eligible Children and Their Parents. Health Policy Online No 15. Washington, D.C: The Urban Institute. http:// www.urban.org/publications/311420.html. Accessed October 13, 2009.

Kenney, G. M., J. Haley, and S. Blumberg. 2002. Awareness and Perceptions of Medicaid and SCHIP Programs among Low-Income Families with Uninsured Children. Findings from Early 2001. Report submitted to the U.S. Department of Health and Human Services, Office of the Assistant Secretary for Planning and Evaluation. (Cited in Holahan, J., L. Dubay, and G.M. Kenney. 2003. Which Children are Still Uninsured and Why. The Future of Children 13(1):55-79.)

Kincheloe, J., J. Frates, and E. R. Brown. 2007. Determinants of Children's Participation in California's Medicaid and SCHIP Programs. Health Services Research 42(2):847-866.

Klerman, J. A., M. Davern, K. T. Call, V. Lynch, and J. D. Ringel. 2009. Understanding the Current Population Survey's Insurance Estimates and the Medicaid "Undercount." Health Affairs 28(6):w991-w1001.

$\mathrm{Ku}, \mathrm{L}$. 2007. Reducing Disparities in Health Coverage for Legal Immigrant Children and Pregnant Women. Washington, D.C.: Center on Budget and Policy Priorities. http://www. cbpp.org/cms/?fa = view\&id=143. Accessed May $1,2009$.

Larson, S. L., and S. C. Hill. 2005. Rural-Urban Differences in Employment-Related Health Insurance. Journal of Rural Health 21(1):21-30.
Lenardson, J. D., E. C. Ziller, A. F. Coburn, and N. J. Anderson. 2009. Profile of Rural Health Insurance Coverage: A Chartbook. Portland, Maine: University of Southern Maine, Muskie School of Public Service, Maine Rural Health Research Center. http://muskie.usm.maine. edu/Publications/rural/Rural-Health-InsuranceChartbook-2009.pdf. Accessed September 15, 2009.

Lewis, K., M. Elwood, and J. Czajka. 1998. Counting the Uninsured: A Review of the Literature. Washington, D.C.: The Urban Institute. http://www.urban.org/publications/ 308032.html. Accessed October 13, 2009.

McAlearney, J. S. 2004. Opportunities for Outreach: Medicaid Participation among Children in Ohio. Journal of Health Care for the Poor and Underserved 15:357-374.

Selden, T. M., J. L. Hudson, and J. S. Banthin. 2004. Tracking Changes in Eligibility and Coverage among Children, 1996-2002. Health Affairs 23(5):39-50.

Selden, T. M., J. S. Banthin, and J. W. Cohen. 1998. Medicaid's Problem Children: Eligible but Not Enrolled. Health Affairs 17(3): 192-200.

Turner, J., M. Boudreaux, and V. Lynch. 2009. A Preliminary Evaluation of Health Insurance Coverage in the 2008 American Community Survey. Washington, D.C.: U.S. Census Bureau. http://www.census.gov/hhes/www/hlthins/ acs08paper/2008ACS_healthins.pdf. Accessed October 16, 2009.

Ziller, E., A. F. Coburn, N. J. Anderson, and S. L. Loux. 2008. Uninsured Rural Families. Journal of Rural Health 24(1):1-11.

Ziller, E., and A. Coburn. 2009. Rural Coverage Gaps Decline Following Public Health Insurance Expansions. Portland, Maine: University of Southern Maine, Muskie School of Public Service, Maine Rural Health Research Center. http://muskie.usm.maine.edu/Publications/rural/ $\mathrm{pb} /$ Rural-Public-Health-Insurance.pdf. Accessed October 13, 2009.

U.S. Department of Health and Human Services. 2009. Secretary Sebelius Awards $\$ 40$ Million to States to Find, Enroll Children in CHIP, Medicaid. Press Release. http://www.hhs.gov/ news/press/2009pres/09/20090930a.html. Accessed October 9, 2008. 